\title{
Chronic renal pain: An approach to investigation and management
}

\author{
Malcolm J. Dewar, MBChB, MMed, MSc, FCUrol(SA); Joseph L. Chin, MD, FRCSC
}

Division of Urology, Western University, London, ON, Canada

Cite as: Can Urol Assoc J 2018;12(6Suppl3):S167-70. http://dx.doi.org/10.5489/cuaj.5327

\section{Introduction}

When chronic flank pain is present, there is most often a cause that is readily identified on routine assessment (Table 1). In the vast majority of cases, resolution of the underlying pathology results in improvement of the pain. Although epidemiological data are sparse, it seems that chronic renal pain syndromes are uncommon. This brief review will address selected conditions in which chronic pain is a primary presenting feature, and give an approach to diagnosis and management.

\section{Autosomal-dominant polycystic kidney disease (ADPKD)}

Chronic renal pain is the most common presenting symptom of ADPKD. A defect of the PKD1 gene on chromosome 16 or the PKD2 gene on chromosome 4 results in the development of multiple renal cysts that begins in childhood and continues progressively throughout life. In addition to pain, affected patients can have hypertension, hematuria, or progressive renal impairment. One helpful clue to the diagnosis of ADPKD is that often family history is positive for the condition. Pain can be caused by infection of cysts, hemorrhage, renal calculi, stretching the renal capsule by mass effect, or torsion of the renal pedicle. Chronic, persistent, mild to moderate pain is most common, although the pain may be severe and debilitating.

Management of pain in these patients should follow a normal, stepwise progression. Tolvaptan is a vasopressin V2-antagonist that has been shown to reduce the growth rate of cysts in ADPKD, as well as reduce the incidence of complications. Invasive treatments specific to ADPKD include cyst aspiration with or without sclerotherapy, laparoscopic cyst de-roofing, renal denervation, or nephrectomy. As with most other chronic pain conditions, invasive treatment is usually only indicated after conservative measures have failed. Cyst aspiration and culture-directed antibiotics are the treatment of choice in this situation. Patients more likely to benefit from surgical treatment of specific cysts are those in whom there is a large symptomatic cyst $(>5 \mathrm{~cm})$ or group of dominant cysts (three cysts $>4 \mathrm{~cm}$ ). Localized tenderness with an ultrasound probe, as well as relief with percutaneous cyst aspiration, might predict an improved response to cyst de-roofing or sclerotherapy. Laparoscopic denervation of the kidney accompanied by nephropexy and renal artery embolization has been described, while laparoscopic nephrectomy can be considered in patients with end-stage renal disease.

\section{Loin pain hematuria syndrome (LPHS)}

LPHS is a very rare condition, characterized by intermittent or persistent flank pain that is often, but not always, accompanied by hematuria. Hematuria, microscopic or macroscopic, appears to be glomerular in origin. Pain can be severe and unrelenting, with a significant impact on quality of life and the ability to function. The clinical syndrome has been well-characterized as follows: a six-month or longer history of flank pain that is either persistently present or occurring in episodes of several days; no superficial or muscular tenderness, but punch tenderness eliciting deep pain is present; microscopic or gross hematuria without clots that often coincides with worsening pain. The pain is usually unilateral, although up to $30 \%$ patients have metachronous bilateral presentations. Synchronous bilateral presentation is rare. The diagnosis is one of exclusion, requiring a negative workup for other recognized urologic and nephrologic causes of flank pain.

These patients are usually younger than 40 years and approximately $70 \%$ are female. One prominent aspect of LPHS is the frequent association with psychiatric and/or psychosocial problems. Among LPHS patients, there is a higher than expected incidence of post-traumatic stress disorder, childhood history sexual or physical abuse, alcohol and other substance abuse, an obligation to care for family members, and employment in healthcare-related occupations. Some have histrionic personality traits. Thus, a formal psychological and/or psychiatric evaluation is strongly recommended to rule out or uncover such underlying concerns. Often, these patients have already had multiple encounters with the healthcare system, seeking treatment for pain. However, with no apparent organic causes for the pain, their complaints may be viewed as contrived and they may be labeled and profiled as drug-seekers. 
The etiology and pathogenesis are unclear, although some patients have a history of glomerular disease (most commonly IgA nephropathy) and up to half have a history of nephrolithiasis. Renal biopsies in patients with LPHS suggests that an abnormality in the glomerular basement membrane (GBM) might be responsible for some of the pathogenesis, since two-thirds of patients have abnormally thin or thick GBMs, and a quarter of patients had significant proteinuria. Ischemic (from renal vascular vasospasm) and auto-immunologic mechanisms have been suggested by some, although not substantiated.

The fact that $30 \%$ of patients have bilateral involvement, either synchronous or metachronous, has a major impact on the management in terms of a goal for nephron preservation. The general management of LPHS is dependent on the severity of pain, narcotic requirement, and extent of disruption of normal daily activity. Among various strategies for renal denervation, renal autotransplantation appears to be the most effective treatment for established LPHS. Even with meticulous patient selection, surgical success rate, defined as long-term pain relief, is approximately $70 \%$. Success of pain relief in renal autotransplantation has mostly been attributed to total denervation, i.e. ,complete severance of nerve supply to the kidney capsule and renal vessels and ureter, and grafting in the iliac fossa (as opposed to the native renal bed). Less successful surgical procedures include renal capsulotomy, in situ denervation. Nephrectomy, with sacrifice of an apparently normally functioning kidney, is less attractive as a primary surgical option. Some physicians have offered the option of nephrectomy as a donation for allograft transplant.

\section{Nephroptosis}

Nephroptosis was first formally described by Joseph Dietl in 1864 (Dietl's crisis). The proposed pathophysiology of the condition involves an abnormal downward or medial mobility of the kidney. Torsion of the renal pedicle or kinking of the ureter leads to episodic flank pain, typically worse when standing and alleviated by lying down. The mechanism of the pain could be transient hydronephrosis, reduced renal perfusion, or traction on the autonomic nerves.

The classic patient is a young, parous female with a low body mass index or history of recent weight loss. The patient typically describes episodic flank pain that is present when standing and is relieved by lying down. The pain usually occurs on the right side, but can be bilateral in approximately onethird of cases. Renal function is typically normal. The diagnosis of nephroptosis is suggested on history and confirmed using specific investigations. Conventional intravenous urography (IVU) performed supine and erect can demonstrate descent of the kidney. Classically, a drop of two vertebral body widths is necessary for the diagnosis. Nuclear renography may also show reduced renal perfusion and urinary obstruction.
For patients with intractable symptoms, some improvement may be obtained by nephropexy (surgical fixation / anchoring of the kidney to minimize its mobility). First described in 1881, this became extremely popular surgical solution for this nephroptosis in the early 1900s, eventually falling out of favour during the latter part of the 20th century. Although traditionally performed via open surgery, nephropexy has seen some resurgence in interest due to improvements in diagnostic studies and refinement of minimally invasive surgical treatments (e.g., laparoscopic approach or even renal fixation include using a percutaneous nephrostomy to cause perinephric fibrosis). Surgery consists of suturing the kidney in several places with non-absorbable suture to adjacent non-mobile tissue (e.g., muscles or peri-ostium of ribs) in an elevated position. When the kidney remains successfully elevated, this treatment hopefully results in decreased obstruction and usually improvement in pain.

\section{General assessment of the patient with apparent chronic renal pain}

The physician must rule out both non-urologic (non-renal) and urologic (renal) causes of flank and abdominal pain (Table 1). Fig. 1 describes the general assessment of the patient with apparent chronic renal pain. The evaluation must include a thorough history of the presenting complaint and general history, as well as focused physical examination. The clinician should try to gauge the impact on quality of life and functioning, as well as the effects on the patient's family. A good social history is also important to identify any potential psychological stressors and to get an understanding of the patient's support network. Aside from basic urinalysis, blood chemistry, and renal ultrasonography, some form of contrast-enhanced axial imaging is essential. For patients with allergy to iodinated contrast or with very poor renal function, uncontrasted computerized tomography (CT) may be of only limited usefulness, in which case magnetic resonance imaging (MRI) (preferably with Gadolinium) scanning might be more helpful. Three-phase contrast-enhanced CT scan will demonstrate the vast majority of vascular, parenchymal, and obstructive causes of pain. Radionuclide renogram can be performed to demonstrate renal hypoperfusion, reduced function, or obstruction, and to calculate differential split renal function, when indicated. Renogram is important to perform when considering surgical options, such as nephrectomy, renal denervation, or renal autotransplantation. CT or MR renal angiography should be considered in suspected LPHS, patients especially when there is substantial haematuria, to rule out vascular anomalies, such as arterio-venous malformations and aneurysms of renal vessels. 


\begin{tabular}{ll}
\hline \multicolumn{2}{l}{ Table 1. Causes of chronic flank pain } \\
\hline Renal & Visceral \\
\hline Parenchymal & Liver \\
Polycystic kidney disease & Gallbladder \\
Glomerulonephritis & Spleen \\
Xanthogranulomatous & Ovary/testis \\
pyelonephritis/pyonephrosis & Pancreas/stomach/aorta* \\
Loin pain-hematuria & Bowel** \\
syndrome & Pulmonary/pleural \\
Renal tumour & Neuropathic \\
Vascular & Shingles/post-herpetic \\
Fibromuscular dysplasia & neuralgia \\
Renal artery aneurysm & Thoracic or lumbar \\
Renal artery dissection & radiculopathy \\
Renal vein thrombosis & Musculoskeletal \\
Perinephric haemorrhage & Costochondritis \\
Left renal vein entrapment & Lower rib pain syndromes \\
(nutcracker syndrome) & Systemic conditions \\
Post-renal/obstructive & Acute intermittent porphyria \\
Nephrolithiasis & Familial Mediterranean fever \\
Retroperitoneal fibrosis & Sickle cell disease \\
Ureteric stricture & Psychiatric \\
Nephroptosis & \\
Fraley's syndrome & \\
\hline *Pain arising from gastric, pancreatic, and aortic pathology tends to be perceived in the \\
midline, but can present as left or right flank pain. **Intestinal pathology more often \\
produces generalized or lower quadrant pain, and usually coexists with other bowel- \\
specific symptoms. & \\
\hline &
\end{tabular}

\section{General management of patient with chronic renal pain}

A multidisciplinary, multimodal team approach involving urology, nephrology, and a specialized chronic pain practitioner is most preferable. The latter physician may offer regional nerve blocks and/or management and monitoring of narcotic analgesic use. A formal psychiatry or clinical psychology consultation is strongly recommended in cases of suspected LPHS. Good support provided by family members, condition-specific support groups, and/or allied health personnel, such as social workers and nurses is invaluable and should be fostered.

Non-pharmacologic methods, such as cognitive behavioural therapy, psychotherapy, physiotherapy, or alternative medicine, might all play a role for these patients, and where appropriate, should be considered early on. Pharmacologic treatment can begin with non-opioid simple analgesics and non-steroidal anti-inflammatories, with the addition of weak opioids and then strong opioids as necessary. Further medications are best initiated by a pain specialist. Options are alpha-2 adrenergic agonists, antidepressants (tricyclics and serotonin norepinephrine reuptake inhibitors [SNRIs]), antiepileptic drugs (gabapentin, pregabalin, and other anticonvulsants), muscle relaxants, $\mathrm{N}$-methyl-d-aspartate (NMDA) receptor antagonists, and topical analgesic agents.

Details of surgical approaches, including minimally invasive ones, to the management of chronic renal pain have been described in the relevant sections for ADPKD, LPHS, and nephroptosis.

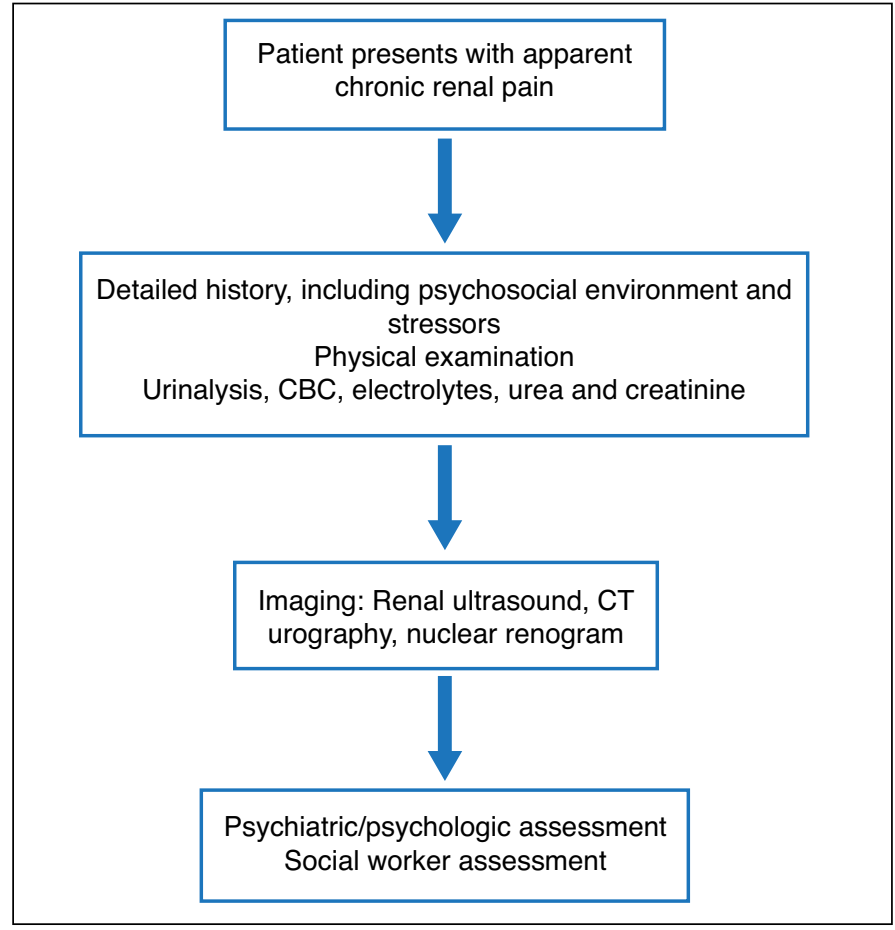

Fig. 1. Flowchart for assessment of chronic renal pain. CBC: complete blood count; CT: computed tomography.

Fig. 2 provides a summary on the management of chronic renal pain.

\section{Summary}

A comprehensive attempt should be made to identify a cause for the chronic flank pain experienced by the patient. In many cases, resolution of the underlying pathology results in improvement of the pain, but for others, pharmacologic, ancillary surgical procedures and psychological support are required to reduce the impact of pain on the patient's quality of life.

Competing interests: Dr. Chin has attended advisory boards for Janssen, Profound Medical Inc., and Sanofi; has received honoraria from Profound Medical Inc.; and has participated in clinical trials supported by AbbVie, Amgen, Janssen, Profound Medical Inc., and US HIFU. Dr. Dewar reports no competing personal or financial conflicts related to this work.

This paper has been peer reviewed.

\section{Recommended reading}

1. Savige J, Tunnicliffe DJ, Rangan GK. KHA-CARI autosomal dominant kidney disease guideline: Management of chronic pain. Semin Nephrol 2015;35:607-11.e3. https://doi.org/10.1016/i. semnephrol.2015.10.013

2. Taba Taba Vakili S, Alam T, Sollinger H. Loin pain hematuria syndrome. Am J Kidney Dis 2014;64:460-72. https://doi.org/10.1053/i.ajkd.2014.01.439 


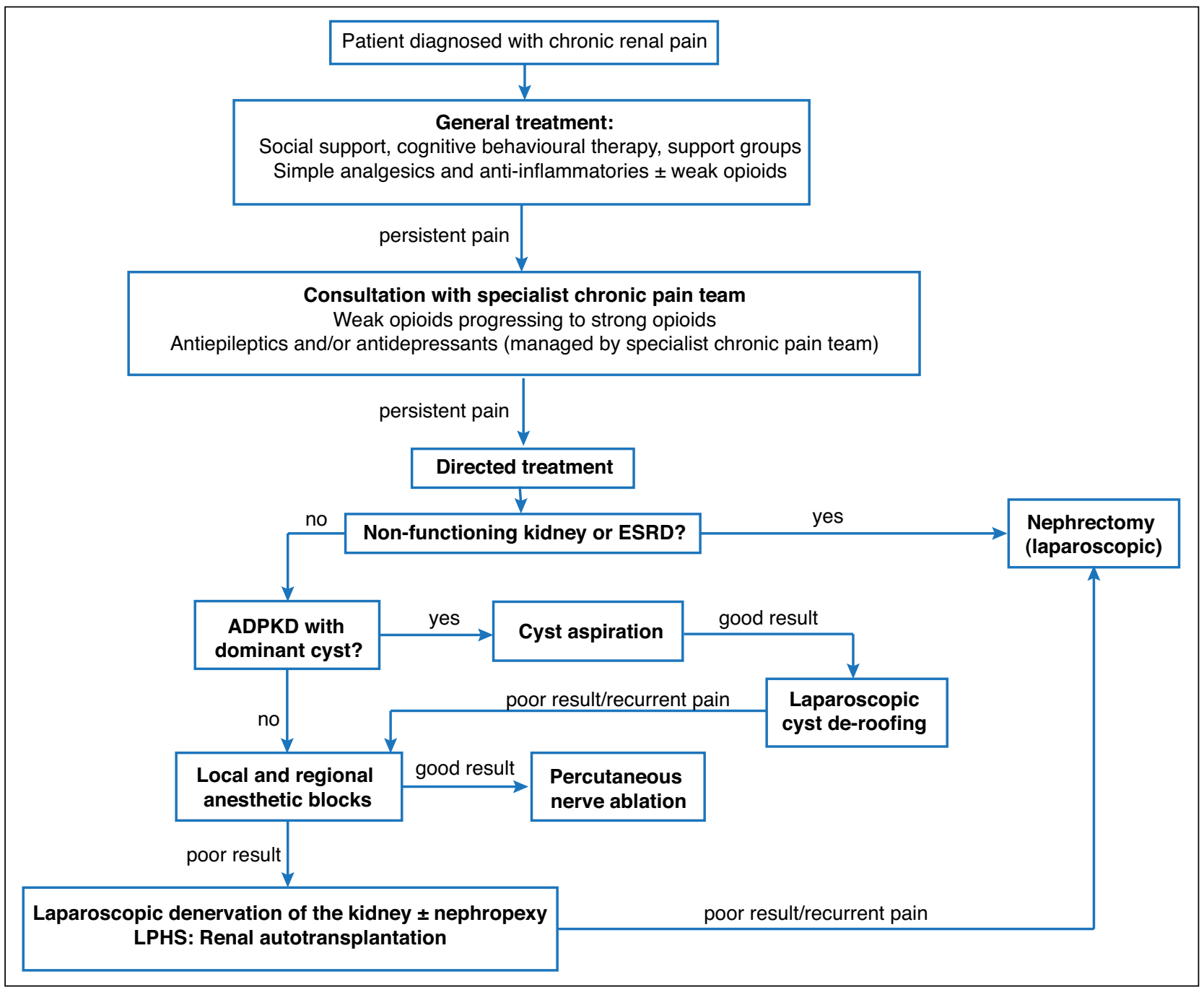

Fig. 2. Management of chronic renal pain. ADPKD: autosomal-dominant polycystic kidney disease; ESRD: end-stage renal disease; LPHS: loin pain-hematuria syndrome.

3. Chin JL, Kloth D, Pautler SE, et al. Renal autotransplantation for the loin pain-hematuria syndrome: Long- term followup of 26 cases. J Urol 1998;160:1232-6. https://doi.org/10.1016/S0022$5347(01) 62503-5$

4. Gözen AS, Rassweiler JJ, Neuwinger F, al. Long-term outcome of laparoscopic retroperitoneal nephropexy. J Endourol 2008;22:2263-7. https://doi.org/10.1089/end.2008.0365
Correspondence: Dr. Malcolm Dewar, Division of Urology, Western University, London, ON, Canada; maldewar@gmail.com 